\title{
Tapping to Carter: A Response to Fischinger and Dyck-Hemming
}

\author{
ÈVE POUDRIER[1] \\ University of British Columbia
}

\begin{abstract}
This short essay responds to issues raised by Fischinger and DyckHemming in their commentary on this author's article "Tapping to Carter: Mensural Determinacy in Complex Rhythmic Sequences.” Borrowing Christopher Hasty's concept of mensural determinacy, I used an excerpt of Elliott Carter's 90+ for piano (1994) as source materials for a tapping experiment aimed at: (1) testing the hypothesis that style-specific expertise correlates with lower tapping variability; (2) exploring the influence of an implied beat on participants' interpretation of the underlying pulse, as shown by spontaneous tapping; and (3) exploring the influence of a subset of musical parameters as well as participants' characteristics on tapping behavior. This response aims to clarify the methodology employed, especially with reference to the interpretation of the results; it also addresses concerns raised by the reviewers in relation to the use of the tapping paradigm to investigate Carter's compositional language. While the experimental method necessarily limited the interpretation of participants' ongoing experience of pulse, the findings provide useful insights on the role of style-specific expertise and call for a more diverse and disciplinarily unbounded methodological approach to the study of musical communication.
\end{abstract}

Submitted 2018 April 24; accepted 2018 April 25.

KEYWORDS: expertise, periodicity, rhythmic complexity, Elliott Carter

\section{INTRODUCTION}

THE repertoire represented by the source materials selected for the current study, namely, twentieth century post-tonal works that exhibit rhythmic layering and metric irregularity, has been largely ignored by experimental research focused on questions of pulse perception. Although this state of affairs may be the result of practical limitations, including experimenters' unfamiliarity with the different musical styles represented, the fact is that it may also have been deemed unfruitful to consider questions of pulse perception in a rhythmic environment characterized by metric flux, especially given the closely-knit relationship that is generally drawn between pulse perception, metric entrainment, and a conception of meter as a nested hierarchy of pulses. Thus, one important goal of the current study was to address this gap, and to do so by using "real" music as source materials. To use actual music as source materials for an experimental study necessarily entails certain methodological choices, which included manipulating the source materials to achieve a balance between ecological validity and experimental control as well as adapting standard performance measures (such as tapping variability and beat synchronization) to address the specific components of pulse perception that were judged to be pertinent to the source materials.

In their commentary, Fischinger and Dyck-Hemming identify three potentially problematic aspects of this study: (1) the relatively low overall synchronization rate and its implications for the interpretation of the results; (2) the absence of a regular beat in Carter's compositional language and the relevance of a perceivable pulse to the composer's aesthetic goals; and (3) the extraction of specific features from the source materials (as opposed to using the undivided whole) combined with the use of mechanical means of production (rather than human performance). Given that the reviewers seem to agree that the loss of some degree of ecological validity may be unavoidable in using experimental methods, and that the manipulations employed in the current study are well represented in previous beat finding experiments using actual music as source materials (e.g., Drake, Penel, \& Bigand, 2000; Snyder \& Krumhansl, 2001; Toiviainen \& Snyder, 2003), my response to their commentary will focus mainly on the first two issues raised. The goal of this 
response is to clarify the methodology used in relation to the research questions and source materials as well as to shed some light on the implications of the findings for future research.

\section{METHODOLOGICAL CONSIDERATIONS}

The three experimental goals of the current study were to: (1) test the hypothesis that style-specific expertise correlates with lower tapping variability; (2) explore the influence of an implied beat on participants' pulse percepts, as shown by spontaneous tapping; and (3) explore the influence of a subset of musical parameters and participants' characteristics on tapping behavior (tapping variability and synchronization). In this section, I review some of the key elements in the experimental design and their relation to these research questions.

As pointed out by Fischinger and Dyck-Hemming, synchronization performance across participants over the 18 trials of the study was relatively low, especially as compared to those observed in previous studies. The specific mean synchronization rates mentioned, $31.9 \%$ and $25.5 \%$, were achieved within the first and third blocks of trials, respectively (and not across the entire experiment), the first featuring the two layers extracted from the source materials separately, without pitch, and the third presenting the same two layers combined (see Table 3, p. 289). It is worth noting that synchronization performance also varied widely across participants: for example, within the first block of trials, tapping synchronization (proportion of taps coinciding with the implied beat) ranged from 15.5 to $70.8 \%(S D=11.5)$. Nonetheless, these measures were not relevant to the main hypothesis being tested, that is, that participants who report a higher level of engagement with twentieth-century music (measured in hours per week listening, analyzing, performing and/or composing such music) exhibit a more stable tapping behavior, that is, a lower overall coefficient of variability, which was calculated independently from synchronization performance (i.e., tapping variability included all recorded taps, whether or not they were synchronized with an implied beat; for more details, see p. 288).

Beyond the issue of style-specific expertise, the purpose of the experiment was to assess the influence of an implied beat (determined based on score analysis and knowledge of the composer's compositional methods) on participants' pulse percepts rather than their ability to identify "the” beat, as is the case in most of the previous beat finding studies. Furthermore, in these studies, the music used as source materials generally features mostly unambiguous beats. In contrast, in Carter's music, not only are there often several contrasting rhythmic layers superposed, but each layer may be based on a different pulse rate and feature only a limited number of isochronous sequences, resulting in a highly irregular rhythmic surface. In this context, the concept of mensural determinacy, borrowed from Christopher Hasty's Meter as Rhythm (1997) becomes especially relevant. As discussed in the article (pp. 278-79), one important aspect of this concept is that it does not assume a stable metric hierarchy, but rather embraces a conception of meter as an emerging phenomenon that arises from the interaction of a listener's attention (with all that this entails, including individual preferences) and a given musical structure.

The current study was thus designed with the assumption that participants may exhibit very different pulse percepts, but that these pulse percepts would emerge in interaction with Carter's beat cues. Previous studies have shown that pulse perception (as represented by tapping period and/or phase) can be influenced by various factors, including level of musical training, individual preferences, and experimental procedure, for example, continuation as compared to spontaneous tapping (Repp, 2005; Repp \& Su, 2013). It has also been shown that familiarity with a given musical style can also have a determining effect on listeners' metric perception (Hannon, Soley, \& Ullal, 2013). To optimize participants' use of acquired listening skills relevant to the source materials and experimental task, all stimuli were listened to once in their entirety before taps were recorded; participants were also instructed to tap to the perceived pulse or beat, which might involve not tapping at all if they felt there was no underlying pulse, or altering the speed or duration associated with the beat if they felt that it was altered, as it may occur in actual music. The two synchronization measures used, tapping and beat synchronization, were based on the percentage of taps that coincided with an implied beat and the percentage of implied beats that were spontaneously synchronized to regardless of the specific tapping profile, and most of the statistical analyses were aimed at testing the significance of observed differences across two conditions (e.g., left-hand vs. right-hand layer, accented vs. unaccented, etc.). The overall low synchronization rate is thus a by-product of the specific task and performance measures used, and cannot be directly compared to previous beat finding studies. 


\section{COMPOSITIONAL CONSIDERATIONS}

As mentioned in the previous section, Carter's music generally exhibits a highly irregular rhythmic surface. Listeners unfamiliar with the composer's style may, at least on a first hearing, find it difficult to disentangle events that have structural significance in the temporal domain from the ongoing flow of rhythmic, melodic and harmonic processes. It is important, however, not to equate methods with intent, and from Carter's own comments, it would appear that the intricate scaffolding of rhythmic layers in his music is more than an intellectual endeavor intended to be understood only by a small number of initiates. In this section, I will first discuss the function of the pulse in Carter's music, with a special emphasis on evidence in support of an experimental investigation of pulse perception in this music. I will then review basic rhythmic characteristics of the source materials pertinent to the interpretation of the results, and discuss the implications of some of the findings for future research.

\section{Pulse Perception in Carter's Music}

From Carter's writings and interviews, it is apparent that the composer writes for a listener who is able not only to recognize the rhetorical function of the different speeds associated with each rhythmic layer, but also to form predictions about future events. Indeed, for Carter, the communication of musical meaning depends crucially on listeners' ability to do these things:

"In my own music, I am keenly aware of the ways in which some of these concepts of time can affect even small details and make them able to participate in larger constructions. For it is the large continuity and conception of progress which determines the choice of all materials in my recent work - any given moment, for the most part, is a bridge from a previous one to a succeeding one and contains both the elements of unexpectedness as well as intelligible relation to the past and anticipation of the future, not always fulfilled in the way anticipated [italics added]." (Bernard, 1997, p. 318)

For Carter, then, musical communication depends on a listener's ability to grasp the relationship between small surface details and underlying rhythmic processes. What is more, the denial of expectations aroused in the listener is an important part of the communicative process.

Despite the irregularities of the rhythmic surface, Carter leaves many clues in the score that can help locate competing beat structures, some of which can be picked up by an "informed" listener, especially if they are highlighted by the performer(s). This is consistent with the composer's aesthetic goals and the particular attributes of his audience (e.g., the likelihood that they are familiar with his compositional language and/or have access to a score, and may listen to a given work multiple times):

[W]hile such works as mine do not always receive performances that present clearly all the materials, their relationships and expressive intentions, still, these are there in the score, and performers and listeners can gradually come to recognize them after successive performances [italics added].” (Bernard, 1997, p. 271)

From these comments, it is reasonable to posit that effective communication in Carter's music may rely on listeners' ability to recognize specific beat cues and willingness to entrain to a pulse that is likely to be playfully challenged. The communicative function of speeds, which result from perceived pulses, with or without a visual aid (such as the composer's score), has also been recognized by several music scholars, and from the published analytical literature (e.g., Capuzzo, 2012; Ravenscroft, 2003; Roeder, 2006; Roeder, 2012), it would seem that much can be gained from engaging Carter's music from the perspective of listeners' rhythmic experience.

\section{The Status of Meter in $90+$}

Before addressing the issue of how the results from this experiment may be used to further our understanding of pulse perception in complex rhythmic structures, it will be useful to clarify some of the concepts traditionally associated with notated meter, and their applicability to Carter's rhythmic structuring. As 
mentioned by Fischinger and Dyck-Hemming, 90+ is based on a slow structural pulse constituted of 90 timespans (the "90+ pulse"). This slow pulse is notated in different ways throughout the piece, depending on how it interacts with other layers resulting from the subdivision of pulses at different speeds. Given the irregularity with which structural pulses are subdivided into faster rhythmic sequences, and the proportional relationship between these concurrent pulses, the characterization of the structural slow pulse as an "obvious short-range meter" is largely untenable (p. 278).

In the section under consideration, the $90+$ pulse (LH) is notated as 8 eighth notes that are in anti-phase with the notated quarter-note beat, and its associated timespans are subdivided in several different ways (Appendix A \& B, pp. 314-15). Concurrently, the contrasting layer (RH) can be related back to an implied beat that coincides with the notated quarter-note beat, but this relationship is camouflaged by faster and varied isochronous sequences with beginning and/or ending events that are frequently out-of-phase with the notated beat and measure. In this context, and as noted in Fischinger and Dyck-Hemming's commentary, the notated meter is merely a performance tool that coordinates the layers. On the other hand, while I agree that the perception of the implied beat in the RH layer is unlikely, a conjecture that seems to be supported by the relatively lower degree of coincidence observed between participants' recorded taps and this implied "quarter-note" beat, Carter's treatment of the 90+ pulse, which is presented as a series of accented events in the lower register throughout the excerpt suggests that the implied whole-note "downbeats" are not merely meant to be experienced through the eyes, as in "the long tradition of 'Augenmusik"” (p. 278).

\section{Pulse Perception and Style-Specific Expertise}

As a music scholar versed in score-based analysis, I may have been contented with the observations briefly sketched in the previous section, and the compelling effect these periodicities had on my own listening experience. However, my search for tangible evidence that can be used to advance our understanding of the perceptual mechanisms at work in the cognition of complex rhythmic sequences demanded that I investigate the observed phenomenon using experimental methods. The results of this study provide further evidence of the important role of style-specific expertise on listeners' readiness to perceive a regular pulse in highly irregular rhythmic sequences, and we may speculate that style-specific expertise may also have played a part in the wide range of synchronization rates observed within the four blocks of trials.

However, rather than providing evidence that "Elliott Carter's music is primarily music for experts" (p. 278), these results, which are based on the proportion of taps that coincided with only one of the secondorder periodicities, suggest that complex rhythmic sequences may give rise to a much wider range of pulse percepts than most of the music that has been experimentally investigated so far, and that future research may benefit from adapting the existing methods to take this into account. In the current study, performance measures were motivated by the specific design of the source materials and Carter's apparent concern for perceptibility. Consequently, meaningful interpretation of synchronization rates can only be obtained by a reference back to the source materials. The significant differences in the proportion of participants' taps that coincided with the implied beat in accented as compared to unaccented rhythmic sequences are consistent with the intuition that rhythmic streaming by means of stress accents may be an effective beat cue not only for performers, but also for listeners. The results also suggest that other factors, such as first-order periodicity, may be highly influential on listeners' beat percepts, and may contribute to, or impede, the effectiveness of these aural cues. Thus, the presence of several successive events with an inter-onset interval corresponding with the implied "quarter-note" beat in the LH layer at the beginning of the excerpt (Figure 1, p. 284) might have been determining for participants' integration of these events into their tapping profile (as attested by a higher proportion of the implied beats synchronized to). On the other hand, irregular subdivision of the notated beat into faster isochronous sequences with longer durations that are out-of-phase in the RH layer was correlated with more tapping variability and significantly lower synchronization rates. It would appear that the use of a "consonant" accentuation pattern against "dissonant" first-order periodicities was an effective way to create layers of a distinctly different temporal quality, and the mismatch between listeners' temporal expectations as represented by their spontaneous tapping attest to the richness of temporal experience that results from an embodied engagement with these source materials.

\section{CONCLUDING REMARKS}

Despite the challenges it entails, the use of actual music as source materials for the exploration of pulse perception using experimental methods has much to offer, especially if it is combined with score-based 
analysis and other methods more generally employed by music scholars. As with any subject matter, methods should be dictated by the research question and not by disciplinary boundaries or aesthetic assumptions. In the current study, the question of the influence of style-specific expertise on tapping variability necessitated the recruitment of participants with a minimum of musical training and varying degrees of exposure to twentieth-century musical styles. Similarly, given that the questions explored pertained to the influence of an implied beat on participants' pulse percepts in a context rich in musical details, each of which could influence participants' percepts locally, it was deemed reasonable to extract the rhythmic structure from its pitch environment and to compare participants' tapping performance to different conditions globally. While this approach necessarily limited the interpretation of participants' on-going experience of pulse, it did afford formal observation of the influence of some significant factors, including accentuation and register. The fact that there was a wide range in the extent to which participants' pulse percepts coincided with the implied (compositional) beat, including at least one participant who achieved near-perfect synchronization, suggests that the investigation of pulse perception in highly irregular rhythmic contexts can provide insight into the influence of style-specific expertise on listening experience. Furthermore, the collection of tapping data specific to a given musical composition affords further exploration of local factors, which could then be tested in more controlled experimental settings.

I am grateful to the reviewers for engaging with the current study and addressing the implications of using experimental methods to investigate questions that ultimately pertain to the larger topic of musical aesthetics and communication. In closing, I would simply like to propose that to subject an excerpt of "real" music to experimental manipulation does not impoverish musical experience, but rather, enriches and diversifies the ways in which we can interact with this music. And Carter? From my limited encounters with him during his last years in New York, my guess is that he would have been amused by this endeavor, and might have interpreted it as yet another fascinating way that human beings interact with one another and with the world in which they co-exist.

\section{ACKNOWLEDGEMENTS}

This article was copyedited by Tanushree Agrawal and layout edited by Kelly Jakubowski.

\section{NOTES}

[1] Correspondence can be addressed to: Dr. Ève Poudrier, University of British Columbia, 6361 Memorial Road, Vancouver, BC V6T 1Z2, Canada, eve.poudrier@ubc.ca.

\section{REFERENCES}

Bernard, J., ed. (1997). Elliott Carter: Collected Essays and Lectures, 1937-1995. Rochester, NY: University of Rochester Press.

Capuzzo, G. (2012). Elliott Carter's “What Next?”: Communication, cooperation, and separation. Rochester, NY: University of Rochester Press.

Drake, C., Penel, A., \& Bigand, E. (2000). Tapping in time with mechanically and expressively performed music. Music Perception, 18(1), 1-23. https://doi.org/10.2307/40285899

Hannon, E. E., Soley, G., \& Ullal, S. (2012). Familiarity overrides complexity in rhythm perception: A crosscultural comparison of American and Turkish listeners. Journal of Experimental Psychology: Human Perception and Performance, 38(3), 543-548. https://doi.org/10.1037/a0027225

Hasty, C. F. (1997). Meter as rhythm. New York: Oxford University Press.

Repp, B. H. (2005). Sensorimotor synchronization: A review of the tapping literature. Psychonomic Bulletin Review, 12(6), 969-992. https://doi.org/10.3758/BF03206433 
Repp, B. H., \& Su, Y. (2013). Sensorimotor synchronization: A review of recent research (2006-2012). Psychonomic Bulletin Review, 20, 403-452. https://doi.org/10.3758/s13423-012-0371-2

Roeder, J. (2006). “Autonomy and dialogue in Elliott Carter’s Enchanted Preludes.” In Analytical Studies in World Music, ed. M. Tenzer, 377-414. New York, NY: Oxford University Press. https://doi.org/10.1093/acprof:oso/9780195177893.003.0012

Roeder, J. (2012). “'The matter of human cooperation’ in Carter's mature style.” In Elliott Carter Studies, eds. M. Boland and J. F. Link, 110-37. Cambridge, U.K.: Cambridge University Press. https://doi.org/10.1017/CBO9781139030755.008

Ravenscroft, B. (2003). "Setting the pace: The role of speeds in Elliott Carter's A Mirror on Which to Dwell." Music Analysis, 22(3), 253-82. https://doi.org/10.1111/j.0262-5245.2003.00186.x

Snyder, J. S., \& Krumhansl, C. L. (2001). Tapping to ragtime: Cues to pulse finding. Music Perception, 18(4), 455-489. https://doi.org/10.1525/mp.2001.18.4.455

Toiviainen, P., \& Snyder, J. S. (2003). Tapping to Bach: Resonance-based modeling of pulse. Music Perception, 21(1), 43. https://doi.org/10.1525/mp.2003.21.1.43 